\title{
Some Entries in Ramanujan's Notebooks
}

\author{
W. Duke
}

\section{Introduction}

Some of Ramanujan's original discoveries about hypergeometric functions and their relation to modular integrals, especially Eisenstein series of negative weight, are still not very well understood. These discoveries take the form of identities that he recorded, without proof, as entries in his notebooks. ${ }^{*}$ In the following sections I will introduce some of these entries, discuss their status, give new proofs of several of them and also provide new results of a similar nature.

\section{Infinite series}

Roughly one hundred years ago, Ramanujan recorded the following identity in his first notebook:

$$
\sum_{\substack{n \geq 1 \\ n \text { odd }}} \frac{1}{n^{2}\left(e^{\frac{n y}{2}}+e^{-\frac{n y}{2}}\right)}=\frac{\sqrt{x}}{4} \frac{1+\left(\frac{2}{3}\right)^{2} x+\left(\frac{2 \cdot 4}{3 \cdot 5}\right)^{2} x^{2}+\left(\frac{2 \cdot 4 \cdot 6}{3 \cdot 5 \cdot 7}\right)^{2} x^{3}+\ldots}{1+\left(\frac{1}{2}\right)^{2} x+\left(\frac{1 \cdot 3}{2 \cdot 4}\right)^{2} x^{2}+\left(\frac{1 \cdot 3 \cdot 5}{2 \cdot 4 \cdot 6}\right)^{2} x^{3}+\ldots} .
$$

Here it is understood that for $0<x<1$,

$$
y=\pi \frac{1+\left(\frac{1}{2}\right)^{2}(1-x)+\left(\frac{1 \cdot 3}{2 \cdot 4}\right)^{2}(1-x)^{2}+\ldots}{1+\left(\frac{1}{2}\right)^{2} x+\left(\frac{1 \cdot 3}{2 \cdot 4}\right)^{2} x^{2}+\left(\frac{1 \cdot 3 \cdot 5}{2 \cdot 4 \cdot 6}\right)^{2} x^{3}+\ldots} .
$$

The basic meaning of this identity can be comprehended by anyone familiar with infinite series. However, as Hardy observed, there is always more in one of Ramanujan's formulæ than meets the eye.

This identity really belongs to the theory of Jacobian elliptic functions. In this theory a central role is played by Legendre's complete

Research supported in part by NSF Grant DMS-0355564.

*See the Notes at the end of the paper for references to the literature. 
elliptic integral of the first kind with modulus $k$ :

$$
K=K(k)=\int_{0}^{\pi / 2}\left(1-k^{2} \sin ^{2} \varphi\right)^{-1 / 2} d \varphi .
$$

Provided $|k|<1$, it is easy to see that $\frac{2}{\pi} K$ can be expressed as a hypergeometric series ${ }^{\dagger}$

$$
\frac{2}{\pi} K=F\left(k^{2}\right)={ }_{2} F_{1}\left(\frac{1}{2}, \frac{1}{2} ; 1 ; k^{2}\right)=1+\left(\frac{1}{2}\right)^{2} k^{2}+\left(\frac{1 \cdot 3}{2 \cdot 4}\right)^{2} k^{4}+\ldots
$$

Following Jacobi, we set $q=e^{-\pi K^{\prime} / K}$, where $K^{\prime}=K\left(k^{\prime}\right)$ and $k^{\prime}=$ $\sqrt{1-k^{2}}$ is the complementary modulus. In terms of the theta constants

$$
\vartheta_{2}=\sum_{n \in \mathbb{Z}} q^{\left(n+\frac{1}{2}\right)^{2}}, \quad \vartheta_{3}=\sum_{n \in \mathbb{Z}} q^{n^{2}} \quad \text { and } \quad \vartheta_{4}=\sum_{n \in \mathbb{Z}}(-1)^{n} q^{n^{2}},
$$

we have Jacobi's fundamental relations

$$
k=\left(\vartheta_{2} / \vartheta_{3}\right)^{2}, \quad k^{\prime}=\left(\vartheta_{4} / \vartheta_{3}\right)^{2} \text { and } \quad F\left(k^{2}\right)=\vartheta_{3}^{2} .
$$

Setting $x=k^{2}$ and $q=e^{-y}$, Ramanujan's identity can be written

$$
4 \sum_{\substack{n \geq 1 \\ n \text { odd }}} \frac{q^{n / 2}}{n^{2}\left(1+q^{n}\right)}=\frac{k_{3} F_{2}\left(1,1,1 ; \frac{3}{2}, \frac{3}{2} ; k^{2}\right)}{F\left(k^{2}\right)} .
$$

The left hand side is recognizable as the Fourier expansion of an Eisenstein series, but of weight -1. The identity (2.2) should be compared with two highlights of Jacobi's Fundamenta Nova. The first is an identity for an Eisenstein series of weight 1:

$$
4 \sum_{\substack{n \geq 1 \\ n \text { odd }}} \frac{q^{n / 2}}{1+q^{n}}=k F\left(k^{2}\right)
$$

Taken together with (2.1), this gives a formula for the number of ways of representing an integer as the sum of two triangular numbers. The second is an identity for an Eisenstein series of weight 0:

$$
4 \sum_{\substack{n \geq 1 \\ n \text { odd }}} \frac{(-1)^{\frac{n-1}{2}} q^{\frac{n}{2}}}{n\left(1+q^{n}\right)}=\arcsin k
$$

${ }^{\dagger}$ Generally we will use the following notation for hypergeometric series

$$
{ }_{m+1} F_{m}\left(a_{1}, \ldots, a_{m+1} ; b_{1}, \ldots, b_{m} ; z\right)=\sum_{n=0}^{\infty} \frac{\left(a_{1}\right)_{n} \ldots\left(a_{m+1}\right)_{n}}{\left(b_{1}\right)_{n} \ldots\left(b_{m}\right)_{n}} \frac{z^{n}}{n !},
$$

where as usual $(a)_{n}=a(a+1) \cdots(a+n-1)=\Gamma(a+n) / \Gamma(a)$. 
This gives a formula for the modular angle that, as Jacobi noted, can be expressed most elegantly as

$$
\frac{1}{4} \arcsin k=\arctan \left(q^{1 / 2}\right)-\arctan \left(q^{3 / 2}\right)+\arctan \left(q^{5 / 2}\right)-\cdots .
$$

While identities of this type for Eisenstein series with nonnegative weights are well understood, those like Ramanujan's in negative weights are not. In this paper I will provide the following new example of weight -2 :

$$
4 \sum_{\substack{n \geq 1 \\ n \text { odd }}} \frac{(-1)^{\frac{n-1}{2}} q^{\frac{n}{2}}}{n^{3}\left(1+q^{n}\right)}=\frac{k k^{\prime}{ }_{4} F_{3}\left(1,1,1,1 ; \frac{3}{2}, \frac{3}{2}, \frac{3}{2} ; 4 k^{2} k^{\prime 2}\right)}{{ }_{3} F_{2}\left(\frac{1}{2}, \frac{1}{2}, \frac{1}{2} ; 1,1 ; 4 k^{2} k^{\prime 2}\right)},
$$

whose analogy with (2.2) is clear. Although he apparently did not discover (2.6), Ramanujan attempted to determine the next case and wrote in his first notebook a statement equivalent to the following:

$$
4 \sum_{\substack{n \geq 1 \\ n \text { odd }}} \frac{q^{n / 2}}{n^{4}\left(1+q^{n}\right)}=\frac{k+\left(\frac{2}{3}\right)^{2}\left[1+\left(\frac{2}{3}\right)^{2}\left\{1+\left(\frac{1}{2}\right)^{2}\right\}\right] k^{3}+\ldots}{F\left(k^{2}\right)^{3}} .
$$

However, Ramanujan drew a very faint line through it and did not record it in his second notebook; its status has remained unclear until now. One can try to guess the rule for the formation of the coefficients, but already the coefficient $\frac{56}{81}$ of $k^{3}$ in the numerator is wrong. Nevertheless, Ramanujan was not too far off; the correct formula reads

$$
\begin{aligned}
4 \sum_{\substack{n \geq 1 \\
n \text { odd }}} \frac{q^{n / 2}}{n^{4}\left(1+q^{n}\right)} & =\frac{k+\frac{76}{81} k^{3}+\cdots+a_{n} k^{2 n+1}+\ldots}{F\left(k^{2}\right)^{3}} \\
\text { where } \quad a_{n} & =\frac{1}{4} \sum_{\ell=0}^{n} \frac{(1)_{\ell}^{2}}{\left(\frac{3}{2}\right)_{\ell}^{2}} \sum_{m=0}^{\ell} \frac{(m+1)_{n-\ell}^{2}}{\left(m+\frac{1}{2}\right)_{n-\ell+1}^{2}} .
\end{aligned}
$$

This last example leads one to suspect that there probably cannot be many more simple hypergeometric identities like (2.2) and (2.6), at least essentially different ones.

As for proofs, Ramanujan did not reveal one for (2.2). It was not until 1991 that a proof was published by Berndt. He deduced it from another entry of Ramanujan from the second notebook that evaluates certain iterated integrals involving hypergeometric series. The proof uses the theory of second order nonhomogeneous differential equations and takes several pages of computation. A very 
short proof of (2.2) as well as a proof of (2.8), both based on continued fractions, are given in $\S 4$. Then (2.6) is proved in $\S 5$ by an extension of Berndt's approach; it follows from a higher order version of Ramanujan's iterated integral. This approach seems to be more general than that by continued fractions and also has applications to the evaluation of integrals of cusp forms in terms of generalized hypergeometric series.

\section{Infinite products}

Before turning to these proofs, consider the following remarkable application Ramanujan found of (2.2), which appears in his lost notebook:

$$
\begin{gathered}
\left\{\left(\frac{1-q^{1 / 2}}{1+q^{1 / 2}}\right)\left(\frac{1+q^{3 / 2}}{1-q^{3 / 2}}\right)^{3}\left(\frac{1-q^{5 / 2}}{1+q^{5 / 2}}\right)^{5} \cdots\right\}^{\log q}\left\{\left(\frac{1-i q^{\prime}}{1+i q^{\prime}}\right)\left(\frac{1+i q^{\prime 2}}{1-i q^{\prime 2}}\right)^{2}\left(\frac{1-i q^{\prime 3}}{1+i q^{\prime 3}}\right)^{3} \cdots\right\}^{2 i \pi} \\
=\exp \left(\frac{\pi^{2}}{4}-\frac{k_{3} F_{2}\left(1,1,1 ; \frac{3}{2}, \frac{3}{2} ; k^{2}\right)}{{ }_{2} F_{1}\left(\frac{1}{2}, \frac{1}{2} ; 1 ; ; k^{2}\right)}\right),
\end{gathered}
$$

where $q=e^{-\pi K^{\prime} / K}$ and $q^{\prime}=e^{-\pi K / K^{\prime}}$. A prototype from Jacobi's classical theory is

$$
\left\{\left(\frac{1+i 1^{1 / 2}}{1-i q^{1 / 2}}\right)\left(\frac{1-i q^{3 / 2}}{1+i q^{3 / 2}}\right)\left(\frac{1+i q^{5 / 2}}{1-i q^{5 / 2}}\right) \cdots\right\}\left\{\left(\frac{1+i q^{\prime / 2}}{1-i q^{1 / 2}}\right)\left(\frac{1-i q^{\prime 3 / 2}}{1+i q^{\prime 3 / 2}}\right)\left(\frac{1+i q{ }^{\prime / 2} / 2}{1-i q^{\prime / 2}}\right) \cdots\right\}=i,
$$

which is a consequence of (2.5) and the obvious modular relation

$$
\arcsin k+\arcsin k^{\prime}=\pi / 2 .
$$

In 2003 Berndt, Chan and Zaharescu observed similarly that (3.1) follows from Ramanujan's identity (2.2) together with the $\tau$-derivative of the modular relation

$$
\sum_{\substack{n \geq 1 \\ n \text { odd }}} \sec \left(\frac{\pi n \tau}{2}\right) n^{-2}=\frac{\pi^{2}}{8}-\tau \sum_{n \geq 1} \chi(n) \tan \left(\frac{\pi n}{2 \tau}\right) n^{-2},
$$

where $q=e^{\pi i \tau}$ and $\chi(n)=(-1)^{(n-1) / 2}$ for odd $n, \chi(n)=0$ otherwise. They also gave a proof of (3.2) using a residue calculation.

It is interesting to note that at the end of his early paper On the integral $\int_{0}^{x} \frac{\tan ^{-1} t}{t} d t$, Ramanujan reveals his technique for obtaining inversion formulas like (3.2) by proving the following:

$$
\frac{\pi}{4} \sum_{n \geq 1} \frac{\operatorname{sech} \pi n x}{n^{2}}=\frac{\pi^{3}}{8}\left(\frac{1}{3}+\frac{x^{2}}{2}\right)-\pi x \sum_{m \geq 1} \frac{\chi(m)}{m^{2}} \operatorname{coth} \frac{m \pi}{2 x} .
$$


He starts with the identity $\pi \operatorname{sech} \pi x=4 \sum_{m \geq 1} \frac{m \chi(m)}{m^{2}+4 x^{2}}$. This gives

$$
\begin{aligned}
& \frac{\pi}{4} \sum_{n \geq 1} \frac{\operatorname{sech} \pi n x}{n^{2}}=\sum_{m, n} \frac{m \chi(m)}{n^{2}\left(m^{2}+4 n^{2} x^{2}\right)} \\
&=\sum_{m \geq 1} \frac{\chi(m)}{m} \sum_{n \geq 1}\left(\frac{1}{n^{2}}-\frac{1}{\left(\frac{m}{2 x}\right)^{2}+n^{2}}\right) \\
&=\frac{\pi^{3}}{8}\left(\frac{1}{3}+\frac{x^{2}}{2}\right)-\pi x \sum_{m \geq 1} \frac{\chi(m)}{m^{2}} \operatorname{coth} \frac{m \pi}{2 x}
\end{aligned}
$$

upon using that $\pi \operatorname{coth} \pi x=\frac{1}{x}+2 x \sum_{n \geq 1} \frac{1}{x^{2}+n^{2}}$ and the evaluations

$$
L(1, \chi)=\frac{\pi}{4}, \quad \zeta(2)=\frac{\pi^{2}}{6} \quad \text { and } \quad L(3, \chi)=\frac{\pi^{3}}{32}
$$

of the Dirichlet $L$-series $L(s, \chi)=\sum_{m \geq 1} \chi(m) m^{-s}$. Note that (3.2) follows easily by combining suitably the identity (3.3) at the arguments $x$ and $x / 2$ and then taking $x=i \tau$. It can also be proved directly by the same method.

A standard calculation using the Lipschitz formula shows that

$$
\frac{d^{2}}{d \tau^{2}} \sum_{\substack{n \geq 1 \\ n \text { odd }}} \sec \left(\frac{\pi n \tau}{2}\right) n^{-2}=\frac{1}{2 \pi} \sum_{\substack{m, n \in \mathbb{Z} \\ n \text { odd }}} \frac{\chi(m)}{(m+n \tau)^{3}}
$$

which is an Eisenstein series of weight 3. It can be seen that (3.2) is simply the transformation of the modular integral of this Eisenstein series as $\tau \mapsto-1 / \tau$ and as such is very well understood in a much more general context. Still, it is of interest to observe that Ramanujan's technique adapts well to more general situations and can be used to prove many of the inversion formulas for the modular integrals that appear in his notebooks. Another relevant example is given by

$$
\sum_{n \geq 1} \chi(n) \sec \left(\frac{\pi n \tau}{2}\right) n^{-3}=\frac{\pi^{3}}{32}\left(\tau^{2}+1\right)-\tau^{2} \sum_{n \geq 1} \chi(n) \sec \left(\frac{\pi n}{2 \tau}\right) n^{-3}
$$

By taking two derivatives of this and combining with (2.6) we obtain another identity like (3.1) but more complicated. 


\section{Continued fractions}

In Ramanujan's first notebook we find a continued fraction that is equivalent to the following:

$$
\frac{4 F}{k} \sum_{\substack{n \geq 1 \\ n \text { odd }}} \frac{q^{n / 2}}{\left(1+q^{n}\right)\left(z F^{2}+n^{2}\right)}=\frac{1}{z+\frac{1^{2}}{1+\frac{2^{2} k^{2}}{z+\frac{3^{2}}{1+\frac{4^{2} k^{2}}{z+\ldots}}}},}
$$

where $F=F\left(k^{2}\right)={ }_{2} F_{1}\left(\frac{1}{2}, \frac{1}{2} ; 1 ; k^{2}\right)$. He rediscovered this beautiful result, which is due to Stieltjes in 1894. It is thus plausible that Ramanujan was aware that it provides a simple proof of (2.2). The even part of the continued fraction is

$$
\frac{1}{z+1^{2}-} \frac{1^{2} 2^{2} k^{2}}{z+2^{2} k^{2}+3^{2}-} \frac{3^{2} 4^{2} k^{2}}{z+4^{2} k^{2}+5^{2}-} \frac{5^{2} 6^{2} k^{2}}{z+6^{2} k^{2}+7^{2}-} \ldots
$$

and this converges for $z=0$ to

$$
\frac{1}{1-} \frac{\left(\frac{2}{3}\right)^{2} k^{2}}{1+\left(\frac{2}{3}\right)^{2} k^{2}-} \frac{\left(\frac{4}{5}\right)^{2} k^{2}}{1+\left(\frac{4}{5}\right)^{2} k^{2}-} \frac{\left(\frac{6}{7}\right)^{2} k^{2}}{1+\left(\frac{6}{7}\right)^{2} k^{2}-} \ldots .
$$

An elementary identity of Euler gives for any complex numbers $a_{1}, \ldots, a_{n}$ that

$$
1+\sum_{\ell=1}^{n} a_{1} a_{2} \cdots a_{\ell}=\frac{1}{1-} \frac{a_{1}}{1+a_{1}-} \frac{a_{2}}{1+a_{2}-} \frac{a_{3}}{1+a_{3}-} \cdots \frac{a_{n}}{1+a_{n}}
$$

It follows easily that the continued fraction in (4.3) equals

$$
1+\left(\frac{2}{3}\right)^{2} k^{2}+\left(\frac{2 \cdot 4}{3 \cdot 5}\right)^{2} k^{4}+\cdots={ }_{3} F_{2}\left(1,1,1 ; \frac{3}{2}, \frac{3}{2} ; k^{2}\right) .
$$

Thus (2.2) follows from (4.1).

Many properties of a continued fraction of the form

$$
f(z)=\frac{1}{z+b_{1}-} \frac{a_{2}}{z+b_{2}-} \frac{a_{3}}{z+b_{3}-} \ldots
$$

are best understood through its relation to the infinite matrix

$$
J=\left(\begin{array}{cccc}
b_{1} & 1 & 0 & \ldots \\
a_{2} & b_{2} & 1 & \ldots \\
0 & a_{3} & b_{3} & \ldots \\
\vdots & & & \ddots
\end{array}\right) .
$$


This is because, as was observed already by Sylvester,

$$
f_{n}(z)=\frac{1}{z+b_{1}-} \frac{a_{2}}{z+b_{2}-} \frac{a_{3}}{z+b_{3}-} \cdots \frac{a_{n}}{z+b_{n}}
$$

is the leading entry of the matrix $\left(z I+J_{n}\right)^{-1}$, where

$$
J_{n}=\left(\begin{array}{cccccc}
b_{1} & 1 & 0 & 0 & \cdots & 0 \\
a_{2} & b_{2} & 1 & 0 & \cdots & 0 \\
0 & a_{3} & b_{3} & 1 & \ldots & 0 \\
\vdots & & & & & \\
0 & 0 & \cdots & a_{n-1} & b_{n-1} & 1 \\
0 & 0 & \cdots & 0 & a_{n} & b_{n}
\end{array}\right) .
$$

A convenient method for computing the power series at $\infty$ of $f(z)$ is to expand $(z I+J)^{-1}$ in a geometric series to get formally

$$
f(z)=z^{-1} \sum_{m=0}^{\infty}(-1)^{m} \mu_{m} z^{-m},
$$

where $\mu_{m}$ is the leading entry of $J_{n}^{m}$ for any $n>m / 2+1$. When applied to $f$ given by (4.2) using

$$
J=\left(\begin{array}{cccc}
1 & 1 & 0 & \cdots \\
1^{2} 2^{2} k^{2} & 2^{2} k^{2}+3^{2} & 1 & \cdots \\
0 & 3^{2} 4^{2} k^{2} & 4^{2} k^{2}+5^{2} & \cdots \\
\vdots & & & \ddots
\end{array}\right)
$$

we get for the Eisenstein series of weight $2 m+1$ the identity

$$
\sum_{\substack{n \geq 1 \\ n \text { odd }}} \frac{n^{2 m} q^{n / 2}}{1+q^{n}}=k \mu_{m} F^{2 m+1},
$$

after expanding the left hand side of (4.1) in a geometric series and matching terms. This gives for $m=0$ another proof of (2.3). In order to obtain an expression for the Eisenstein series of negative weight $-2 m-1$, we need to compute $f^{(m)}(0)$.

Returning to the general $f$, we have from (4.5) that for $m \geq 0$

$$
(-1)^{m} f_{n}^{(m)}(0) / m \text { ! }
$$

is the leading entry of $J_{n}^{-m-1}$. Write $d_{n}=\operatorname{det} J_{n}$. Turnbull showed that the $i j$-th entry of $J_{n}^{-1}$ can be expressed in terms of $f$ by

$$
(-1)^{i+j} \frac{d_{i-1} d_{j-1}}{a_{1} \cdots a_{j}}\left(f_{n}(0)-f_{r}(0)\right), \quad \text { where } \quad r=\max (i-1, j-1),
$$


when $a_{1}=d_{0}=1$ and $f_{0}=0$. It follows that

$$
-f_{n}^{\prime}(0)=f_{n}(0)^{2}+\sum_{j=1}^{n-1} \frac{d_{j}^{2}}{a_{1} a_{2} \ldots a_{j+1}}\left(f_{n}(0)-f_{j}(0)\right)^{2} .
$$

Suppose now that $c_{j} \neq 0$ and that $f_{n}(z)$ has the form

$$
\begin{aligned}
f_{n}(z) & =\frac{1}{z+} \frac{c_{1}}{1+} \frac{c_{2}}{z+} \frac{c_{3}}{1+} \frac{c_{4}}{z+\cdots \frac{c_{2 n-1}}{1}} \\
& =\frac{1}{z+c_{1}-} \frac{c_{1} c_{2}}{z+c_{2}+c_{3}-} \frac{c_{3} c_{4}}{z+c_{4}+c_{5}-} \ldots \frac{c_{2 n-3} c_{2 n-2}}{z+c_{2 n-2}+c_{2 n-1}} .
\end{aligned}
$$

We have by (4.4) again that

$$
f_{n}(0)=\frac{1}{c_{1}}+\frac{c_{2}}{c_{1} c_{3}}+\cdots+\frac{c_{2} c_{4} \cdots c_{2 n-2}}{c_{1} c_{3} \cdots c_{2 n-1}} .
$$

Furthermore in this case $d_{j}=c_{1} c_{3} \cdots c_{2 j-1}$, so Turnbull's identity (4.9) yields the explicit formula

$$
-f_{n}^{\prime}(0)=f_{n}(0)^{2}+\sum_{j=1}^{n-1} \frac{c_{1} c_{3} \cdots c_{2 j-1}}{c_{2} c_{4} \cdots c_{2 j}}\left(f_{n}(0)-f_{j}(0)\right)^{2},
$$

where

$$
f_{n}(0)-f_{j}(0)=\frac{c_{2} c_{4} \cdots c_{2 j}}{c_{1} c_{3} \cdots c_{2 j+1}}+\cdots+\frac{c_{2} c_{4} \cdots c_{2 n-2}}{c_{1} c_{3} \cdots c_{2 n-1}} .
$$

In order to prove (2.8) we apply (4.10) to the $n$th convergent of the even part (4.2) of Stieltjes continued fraction and take the limit as $n \rightarrow \infty$. This leads to the identity

$$
\frac{4 F^{3}}{k} \sum_{\substack{n \geq 1 \\ n \text { odd }}} \frac{q^{n / 2}}{n^{4}\left(1+q^{n}\right)}=\sum_{\ell=0}^{\infty} \frac{\left(\frac{3}{2}\right)_{\ell}^{2}}{(1)_{\ell}^{2}} \frac{1}{(2 \ell+1)^{2}}\left[\sum_{n=\ell}^{\infty} \frac{(1)_{n}^{2}}{\left(\frac{3}{2}\right)_{n}^{2}} k^{2 n-2 \ell}\right]^{2} k^{2 \ell} .
$$

After some manipulation this can be put into the form (2.8).

Another continued fraction found by Ramanujan can be written

$$
\begin{aligned}
& \frac{4}{k k^{\prime}} \sum_{\substack{n \geq 1 \\
n \text { odd }}} \frac{(-1)^{\frac{n-1}{2}} q^{\frac{n}{2}} n}{\left(1+q^{n}\right)\left(z F^{2}+n^{2}\right)} \\
& \quad=\frac{1}{z+\left(1-2 k^{2}\right)-} \frac{2^{2}\left(1-2^{2}\right)\left(k k^{\prime}\right)^{2}}{z+3^{2}\left(1-2 k^{2}\right)-} \frac{4^{2}\left(1-4^{2}\right)\left(k k^{\prime}\right)^{2}}{z+5^{2}\left(1-2 k^{2}\right)-\ldots} .
\end{aligned}
$$


We will need below the following easy consequence of (4.11) and (4.6):

$$
4 \sum_{\substack{n \geq 1 \\ n \text { odd }}} \frac{(-1)^{\frac{n-1}{2}} n^{3} q^{\frac{n}{2}}}{1+q^{n}}=k k^{\prime}\left(1-2 k^{2}\right) F^{4} .
$$

Using Turnbull's identity (4.9) as above on (4.11) together with (2.6) gives an (apparently complicated) hypergeometric identity. It would be interesting to derive (2.6) by proving this identity directly. However, I will proceed differently.

\section{Iterated integrals}

Ramanujan's notebooks contain an unusual iterated integral involving a general Gauss hypergeometric series

$$
F(x)={ }_{2} F_{1}(a, b ; c ; x) .
$$

Assuming that $r>1$ this identity can be written ${ }^{\ddagger}$

$$
F(x) \int_{0}^{x} \int_{0}^{u} t^{r-2} F(t) d t \frac{d u}{\omega(u)}=\frac{x^{r-c}(1-x)^{1-d}}{(r-1)(r-c)}{ }_{3} F_{2}\left[\begin{array}{c}
r-a, r-b, 1 \\
r, r-c+1
\end{array} ; x\right],
$$

where $d=a+b-c+1$ and

$$
\omega(x)=\omega(a, b, c ; x)=x^{c}(1-x)^{d} F^{2}(x) .
$$

It seems likely that Ramanujan considered this to be an identity between power series. His method for deriving it is unknown. If we assume that $c=a+b+1 / 2$ we can give a three-fold version as well. For $r>1$ we have

$$
\begin{aligned}
F^{2}(x) \int_{0}^{x} \int_{0}^{v} \int_{0}^{u} t^{r-2} F^{2}(t) d t \frac{d u}{\omega(u)} \frac{d v}{\omega(v)} \\
=\frac{x^{r+1-2 c}}{(r-1)(r-c)(r-2 c+1)}{ }_{4} F_{3}\left[\begin{array}{c}
r-2 a, r-2 b, r-a-b, 1 \\
r, r-c+1, r-2 c+2
\end{array} ; x\right],
\end{aligned}
$$

where $\omega(x)=x^{c}(1-x)^{1 / 2} F^{2}(x)$.

In the special case $c=1, r=3 / 2$ and $a=b=1 / 2,(5.1)$ is equivalent to (2.2). This is seen by making in both integrals variable changes of the form $y=\pi F(1-t) / F(t)$, for which $\frac{d y}{d t}=-\omega(t)^{-1}$,

\footnotetext{
${ }^{\ddagger}$ In this section the parameters $a, b, c$ of $F(x)$ may change and must be understood from the context in which $F(x)$ appears. We shall suppose for simplicity that $a, b>0$ and that $0<c \leq 1$. As usual, the range of these parameters in an identity can be extended by analytic continuation.
} 
and using the identity of Jacobi:

$$
4 \sum_{\substack{n \geq 1 \\ n \text { odd }}} \frac{(-1)^{\frac{n-1}{2}} n^{2} q^{\frac{n}{2}}}{1-q^{n}}=k^{2} k F^{3}\left(k^{2}\right) .
$$

Similarly, when $a=b=1 / 4,(5.2)$ is equivalent to (2.6). To see this, make in each integral a change of variable of the form

$$
t=4 k^{2} k^{2}=4 k^{2}\left(1-k^{2}\right),
$$

for which

$$
\frac{d t}{d y}=-4 k^{2} k^{\prime 2}\left(1-2 k^{2}\right)_{2} F_{1}\left(\frac{1}{2}, \frac{1}{2} ; 1 ; k^{2}\right)=-\omega\left(4 k^{2} k^{\prime 2}\right),
$$

after applying the Gauss quadratic transformation

$$
{ }_{2} F_{1}\left(\frac{1}{2}, \frac{1}{2} ; 1 ; k^{2}\right)={ }_{2} F_{1}\left(\frac{1}{4}, \frac{1}{4} ; 1 ; 4 k^{2} k^{2}\right)=F\left(4 k^{2} k^{2}\right) .
$$

Next execute the integrals using the identity

$$
4 \sum_{\substack{n \geq 1 \\ n \text { odd }}} \frac{(-1)^{\frac{n-1}{2}} n^{3} q^{\frac{n}{2}}}{1+q^{n}}=k k^{\prime}\left(1-2 k^{2}\right) F^{4}\left(4 k^{2} k^{2}\right),
$$

which follows from (4.12) and (5.4). Finally, apply Clausen's identity

$$
F^{2}(x)={ }_{3} F_{2}\left(\frac{1}{2}, \frac{1}{2}, \frac{1}{2} ; 1,1 ; x\right) .
$$

Berndt was the first to provide a proof of Ramanujan's formula (5.1) and to use it to derive (2.2). We now indicate a generalization and simplification of his proof of (5.1) that also gives a proof of (5.2), hence (2.6). Recall that $F$ solves the hypergeometric equation $L F=$ 0 , where

$$
L=D_{x}^{2}+\frac{c-(a+b+1) x}{x(1-x)} D_{x}-\frac{a b}{x(1-x)},
$$

which can also be written as

$$
x(1-x) L=\delta(\delta+c-1)-x(\delta+a)(\delta+b),
$$

when $\delta=x D_{x}$. Taking $x \mapsto 1-x$ shows that

$$
G(x)=\frac{\Gamma(a) \Gamma(b)}{\Gamma(c) \Gamma(d)}{ }_{2} F_{1}(a, b ; d ; 1-x)
$$

is also a solution and a standard calculation on hypergeometric series shows that again

$$
\left(\frac{G(x)}{F(x)}\right)^{\prime}=-\omega(x)^{-1} .
$$


For any nonnegative integer $m$ let $L^{(m)}$ be the unique monic linear differential operator of order $m+1$ that satisfies $L^{(m)} F^{j} G^{m-j}=0$ for each $j=0, \ldots, m$. Further let

$$
\mathcal{F}_{m}(x)=\frac{1}{m !} \int_{0}^{x}\left(\frac{G(t)}{F(t)}-\frac{G(x)}{F(x)}\right)^{m} t^{r-2} F^{m}(t) d t .
$$

It can be checked that

$$
\left(\omega(x) D_{x}\right)^{m+1} \mathcal{F}_{m}(x)=x^{c+r-2}(1-x)^{d} F^{m+2}(x),
$$

while for $0 \leq \ell \leq m$

$$
\left.\left(\omega(x) D_{x}\right)^{\ell} \mathcal{F}_{m}(x)\right|_{x=0}=0 .
$$

Since we have that

$$
F^{m}(x) \mathcal{F}_{m}(x)=\frac{1}{m !} \int_{0}^{x}(G(t) F(x)-G(x) F(t))^{m} t^{r-2} d t
$$

it also follows that

$$
L^{(m)} F^{m} \mathcal{F}_{m}=x^{r-m c-2}(1-x)^{-m d} .
$$

To prove (5.1), suppose that $m=1$ and observe that it follows from its power series expansion that

$$
w=\frac{x^{r-1}}{(r-1)(r-c)}{ }_{3} F_{2}\left[\begin{array}{c}
r-a, r-b, 1 \\
r, r-c+1
\end{array} ; x\right]
$$

satisfies the nonhomogeneous hypergeometric equation

$$
(\delta(\delta+1-c)-x(\delta+1-a)(\delta+1-b)) w=x^{r-2} .
$$

Using this a calculation shows that

$$
L\left(x^{1-c}(1-x)^{1-d} w\right)=x^{r-c-2}(1-x)^{-d} .
$$

From (5.8) we have that

$$
\mathcal{F}_{1}(x)=\int_{0}^{x} \int_{0}^{u} t^{r-2} F(t) d t \frac{d u}{\omega(u)},
$$

while by (5.10), $L\left(F \mathcal{F}_{1}\right)=x^{r-c-2}(1-x)^{-d}$, so from (5.11)

$$
\mathcal{F}_{1}(x)=\frac{x^{r-c}(1-x)^{1-d}}{(r-1)(r-c) F(x)}{ }_{3} F_{2}\left[\begin{array}{c}
r-a, r-b, 1 \\
r, r-c+1
\end{array} ; x\right]+c_{1}+c_{2} \frac{G(x)}{F(x)}
$$

for constants $c_{1}, c_{2}$. Applying $\omega(x) D_{x}$ to both sides and using (5.6) and (5.9), we see that $c_{2}=0$. Taking $x=0$ we then see that $c_{1}=0$, giving (5.1). Turning now to the proof of (5.2), when $c=a+b+1 / 2$ the symmetric square $L^{(2)}$ of $L$ is hypergeometric; we have $x^{3}(1-x) L^{(2)}=\delta(\delta+2 c-2)(\delta+c-1)-x(\delta+2 a)(\delta+2 b)(\delta+a+b)$.

As before, it follows from the power series that

$$
L^{(2)} w=x^{r-2 c-2}(1-x)^{-1}
$$


where $w$ denotes the right hand side of (5.2). From (5.8) we have that

$$
\mathcal{F}_{2}(x)=\int_{0}^{x} \int_{0}^{v} \int_{0}^{u} t^{r-2} F^{2}(t) d t \frac{d u}{\omega(u)} \frac{d v}{\omega(v)},
$$

while by (5.10), $L^{(2)} F^{2} \mathcal{F}_{2}=x^{r-2 c-2}(1-x)^{-1}$. Thus we have

$$
\begin{aligned}
\mathcal{F}_{2}(x)=\frac{x^{r+1-2 c}}{(r-1)(r-c)(r-2 c+1) F^{2}(x)}{ }_{4} F_{3}\left[\begin{array}{c}
r-2 a, r-2 b, r-a-b, 1 \\
r, r-c+1, r-2 c+2
\end{array} ; x\right] \\
+c_{1}+c_{2} \frac{G(x)}{F(x)}+c_{3}\left(\frac{G(x)}{F(x)}\right)^{2},
\end{aligned}
$$

for constants $c_{1}, c_{2}$ and $c_{3}$. Again we can conclude that $c_{3}=c_{2}=c_{1}=$ 0 using (5.6) and (5.9), thus proving (5.2). This also completes the proof of the identity (2.6).

It should now be clear that one may compute for any positive integer $m$ the $(m+1)$-fold $d t / \omega$-integral of $t^{r+c-2}(1-t)^{d} F^{m+2}(t)$, or even of $t^{r+c-2}(1-t)^{s} F^{m+2}(t)$ for more general $s$, in terms of solutions to a nonhomogeneous linear ODE of order $m+1$. However, such solutions will only rarely be hypergeometric. A non-hypergeometric example considered by Ramanujan that can easily be treated in this manner when $m=1$ is given by the identity

$$
4 \sum_{\substack{n \geq 1 \\ n \text { odd }}} \frac{(-1)^{\frac{n-1}{2}} q^{\frac{n}{2}}}{n^{2}\left(1-q^{n}\right)}=\frac{k}{F} \sum_{n=0}^{\infty} \frac{(n !)^{2}}{\left(\frac{3}{2}\right)_{n}^{2}} \sum_{\ell=0}^{n} \frac{\left(\frac{1}{2}\right)_{\ell}^{2}}{(\ell !)^{2}} k^{2 n},
$$

when $a=b=1 / 2$ and $c=1$. A more difficult case would be (2.8), where one would have to take $m=3$.

On the other hand, if we leave the realm of Eisenstein series there are examples of cusp forms of weights 3 and 4 that have hypergeometric integrals. For instance, define the integers $a_{n}$ by

$$
q \prod_{n \geq 1}\left(1-q^{4 n}\right)^{6}=\sum_{n \geq 1} a_{n} q^{n} .
$$

Then it follows from (5.1) when $r=5 / 4$ and (2.1) that

$$
\sum_{n \geq 1} a_{n} q^{\frac{n}{4}} n^{-2}=\frac{\sqrt{k}_{3} F_{2}\left(\frac{3}{4}, \frac{3}{4}, 1 ; \frac{5}{4}, \frac{5}{4} ; k^{2}\right)}{2 F\left(k^{2}\right)} .
$$

Now $\sum_{n>1} a_{n} e(n \tau)$ is the Fourier expansion of the unique newform of weight 3 for $\Gamma_{0}(16)$ with character $\chi$ defined below (3.2). Here, as usual, we write $e(z)=e^{2 \pi i z}$. Similarly, there is a newform of weight 4 for $\Gamma_{0}(64)$ whose Fourier expansion begins

$$
\sum_{n \geq 1} b_{n} e(n \tau)=e(\tau)-22 e(5 \tau)-27 e(9 \tau)+18 e(13 \tau)-94 e(17 \tau)+\ldots
$$


and for which we can deduce from (5.2) that

$$
\sum_{n \geq 1} b_{n} q^{\frac{n}{4}} n^{-3}=\frac{\sqrt{k k^{\prime}}{ }_{4} F_{3}\left(\frac{3}{4}, \frac{3}{4}, \frac{3}{4}, 1 ; \frac{5}{4}, \frac{5}{4}, \frac{5}{4} ; 4 k^{2} k^{\prime 2}\right)}{2{ }_{3} F_{2}\left(\frac{1}{2}, \frac{1}{2}, \frac{1}{2} ; 1,1 ; 4 k^{2} k^{\prime 2}\right)} .
$$

The curious reader may find some other interesting examples when the monodromy group of $F(a, b ; c ; x)$ is an arithmetic hyperbolic triangle group.

Acknowledgments: I thank Bruce Berndt and the referee for a number of helpful suggestions.

\section{Notes}

\section{$\S 1$. Introduction}

According to Watson [27] and Berndt [6], Ramanujan must have recorded most of the entries of his first notebook from about 1904 until about 1913. The second notebook is a revised and enlarged version of the first, likely written between 1913 and 1914, before he left for England. Both were published, along with a third notebook, in facsimile form and in two volumes, in 1957 [20]. The first notebook is in volume I while the second and third are in volume II. The manuscript now known as his lost notebook was likely written between 1919 and 1920 [1, p.1] and was published in [21].

\section{$\S 2$. Infinite series}

Ramanujan's identity appears in [20, I p. 280 and II p. 218]. Hardy's remark is in his obituary notice for Ramanujan, reproduced in [19, footnote on p. XXV].

A standard reference for Jacobi's theory is [29] and one for (generalized) hypergeometric series is [4]. For expositions of Ramanujan's work on hypergeometric series see Lecture VII of Hardy's book [14], which is based on [13], and the more recent and comprehensive accounts [2] and [5, II]. Jacobi's formulas (2.3-2.5) are in $[16,(6)$ p.159, (46),(47) p.164]. Ramanujan also entered these in his notebooks: see [20, II p. 215]. His exposure to the theory of elliptic functions before he came to England is discussed in [8].

Ramanujan's crossed out entry is in [20, I p.280] and is discussed in [5, V p.402].

Berndt's proof of (2.2) is in [5, III p.153].

\section{$\S 3$. Infinite products}

Ramanujan's infinite product identity (3.1) appears in [21, p. 209]. The original entry, which is nearly illegible, was deciphered by Berndt and its derivation from (2.2) appears in [7].

Ramanujan's paper is \#7 in [19, p.40].

The inversion formula (3.4) is a special case of an entry found in [20, I p.279]. See also [5, II p.276]. 


\section{$\S 4$. Continued fractions}

Ramanujan's continued fraction is in [20, I p.156, II p.220] and also in his first letter to Hardy [19, p.350]. Stieltjes result can be found in [23, p. 555 and p.733]. Rogers [22] independently found an equivalent continued fraction. An improvement on Rogers's treatment is given in [10]. That Ramanujan's version follows from the work of Rogers was pointed out by Preece [18]. See also [5, III p.163]. For a proof of (4.4) see [26, p.17].

For Sylvester's contribution see [24]. A very readable account of the connection between continued fractions and matrices was given by Whittaker [28]. Hermite [15] gave another method to compute the identity (4.7) for Eisenstein series of positive weight based on the Tayler expansion of the elliptic function cn (see also [12, p.252]). See also the recent work of Milne [17].

Turnbull's results (4.8) and (4.9) are proved in [25, I $\S 3, \S 4]$.

The continued fraction (4.11) is found in [20, I p.156 and II p.220]. For a proof see [5, III p.166]. The identity (4.12) also appears in [20, I p.275 and II p.214] and is proved in [5, III p.135].

\section{$\S 5$. Iterated integrals}

Ramanujan's identity (5.1) appears in [20, I p.236 and II p.139], the integrals being indefinite. The definite version is from [5, II p.88]. Another formulation in case $a=b=1 / 2$ and $c=1$ is found in [20, II p.210].

Jacobi's formula (5.3) is found in [16, (43) p.164]. For Gauss's quadratic transformation and Clausen's identity see, for instance, [4, p.95, p.86]. The special case of Clausen's identity (5.5) appears with proof in Gauss's Nachlass [11, III p.424.].

Berndt's proof of (5.1) is in [5, II p.88].

A useful reference for the theory of nonhomogeneous linear differential equations is [3]. See Chapter 6 for the theory of nonhomogeneous hypergeometric functions and page 277 for a discussion of nonhomogeneous generalized hypergeometric functions.

Ramanujan's identity (5.13) is in [20, I p. 280], directly above the crossed out entry (2.7). A proof of (5.13) can be found in [5, V p.399]. Some interesting results about $L^{(3)}$ can be found in [9].

\section{References}

[1] G.E. Andrews and B.C. Berndt, Ramanujan's lost notebook. Part I. Springer, New York, 2005.

[2] R. Askey, Ramanujan and hypergeometric and basic hypergeometric series. Ramanujan: essays and surveys, 277-324, Hist. Math., 22, AMS Providence, 2001.

[3] A.W. Babister, Transcendental functions satisfying nonhomogeneous linear differential equations. Macmillan, New York 1967

[4] W.N. Bailey, Generalized hypergeometric series. Cambridge Tracts in Mathematics and Mathematical Physics, No. 32 Stechert-Hafner, Inc., New York 1964

[5] B.C Berndt, Ramanujan's notebooks. Parts I-V. Springer-Verlag, New York 19851998 
[6] B.C. Berndt, An overview of Ramanujan's notebooks, Ramanujan: essays and surveys, 143-164, Hist. Math., 22, Amer. Math. Soc., Providence, RI, 2001.

[7] B.C. Berndt, H.H. Chan and A. Zaharescu, A quasi-theta product in Ramanujan's lost notebook. Math. Proc. Cambridge Philos. Soc. 135 (2003), no. 1, 11-18.

[8] B.C. Berndt and R.A. Rankin, The books studied by Ramanujan in India. Amer. Math. Monthly 107 (2000), no. 7, 595-601.

[9] T.W. Chaundy, On Clausen's hypergeometric identity. Quart. J. Math. Oxford Ser. (2) 91958 265-274.

[10] P. Flajolet and J. Franon, Elliptic functions, continued fractions and doubled permutations. European J. Combin. 10 (1989), no. 3, 235-241.

[11] C. F. Gauss, Werke, Göttingen and Leipzig, 1863-1927.

[12] H. Hancock, Lectures on the theory of elliptic functions,I. Wiley, 1910.

[13] G.H. Hardy, A chapter from Ramanujan's notebook, Proc. Cambridge Phil. Soc. 21, (1923) 492-503.

[14] G.H. Hardy, Ramanujan. Twelve lectures on subjects suggested by his life and work, Third ed. Chelsea, New York 1978.

[15] C. Hermite, Remarques sur le développment de $\cos$ am $x$, C.R Acad. Sci. LVII 1863,613 in Oeurves II p.264.

[16] C.G.J. Jacobi, Fundamenta Nova, in Werke I, Berlin, 1891 (reprinted by Chelsea, New York 1969.

[17] S. C. Milne, Infinite families of exact sums of squares formulas, Jacobi elliptic functions, continued fractions, and Schur functions. Ramanujan J. 6 (2002), no. 1, 7-149.

[18] C.T. Preece, Theorems stated by Ramanujan. VI: Theorems on continued fractions. Journ. L. M. S. 4, 34-39. 1929

[19] S. Ramanujan, Collected Papers, Chelsea, New York, 1962.

[20] S. Ramanujan, Notebooks (2 volumes), Tata Institute of Fundamental Research, Bombay, 1957.

[21] S. Ramanujan, The lost notebook and other unpublished papers. Narosa Publishing House, New Delhi, 1988.

[22] L.J. Rogers, On the representation of certain asymptotic series as convergent continued fractions. Proc. Lond. M. S. 4, 72-89. (1906)

[23] T.J. Stieltjes, Recherches sur les fractions continues, in Euvres complétes/Collected papers. Vol. II. Reprint of the 1914-1918 edition. Springer-Verlag, Berlin, 1993.

[24] J. J. Sylvester, On a fundamental rule in the algorithm of continued fractions, Phil. Mag. 6, 297-299, 1853 in Mathematical Papers I , 641.

[25] H.W. Turnbull, Matrices and continued fractions. I, II. Proceedings Royal Soc. Edinburgh 53; 151-163, 208-219. (1933)

[26] H.S. Wall, Analytic Theory of Continued Fractions. D. Van Nostrand Company, Inc., New York, N. Y., 1948.

[27] G.N. Watson, Ramanujan's note books. Journal L. M. S. 6, 137-153. 1931

[28] E.T. Whittaker, On the theory of continued fractions. J. Edinb. R. S. Proc. 36, 243255. (1915)

[29] E.T. Whittaker and G.N. Watson, A course of modern analysis. Fourth edition. Cambridge University Press, New York 1962

UCLA MATHematics DePT., Box 951555, Los ANGELES, CA 90095, USA

E-mail address: wdduke@ucla.edu 